REFLEXIONES ACERCA DE LA DEMOCRACIA, CIUDADANÍA Y EDUCACIÓN EN AMÉRICA LATINA

LUCILA REYES SARMIENTO

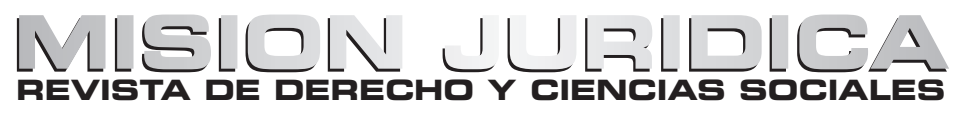





\title{
REFLEXIONES ACERCA DE LA DEMOCRACIA, CIUDADANÍA Y EDUCACIÓN EN AMÉRICA LATINA*
}

\author{
REFLECTIONS ON DEMOCRACY, \\ CITIZENSHIP AND EDUCATION \\ IN LATINAMERICA
}

\author{
REFLEXÓES SOBRE A DEMOCRACIA, \\ A CIDADANIA E A EDUCAÇÃ́o \\ NA AMÉRICA LATINA
}

\author{
LUCILA REYES SARMIENTO* \\ lucinkarey2001@yahoo.es \\ Universidad Nacional de Colombia \\ Recibido par académico: 15-04-2011. Aceptado: 27-07-2011
}

\begin{abstract}
"A Mercurio, hijo de Júpiter y Maya, se le representa juvenil y grácil, dotado de pequeñas alas en los tobillos y vestido con ligero manto. Porta en su mano derecha el caduceo (varita encantada) y en la izquierda la bolsa del dinero -roba a dioses humanos, engaña, espía, soborna, lleva las almas al infierno y las devuelve a la tierra-. Se hace adorar por los pastores y venerar por los atletas, pero por sobre todo es deidad de comerciantes. De enfant terrible del Olimpo se torna con los hados en servil comunicador de los dioses y siniestro ejecutor de sus designios. Por órdenes de Júpiter, encadenó a Prometeo, el titán amigo de los hombres, el que les enseñó a razonar, cultivar, edificar y domesticar". Pedro Agustín Díaz Arenas, Relaciones internacionales de dominación. Fases y facetas [1998].
\end{abstract}

\section{RESUMEN}

Este artículo reflexiona acerca de los significados de ciudadanía y democracia y el papel que juega la educación en la construcción de democracia e identidad en América Latina, una sociedad históricamente desigual. Ser ciudadano y vivir en una democracia es más que ejercer el derecho al voto. La globalización en su estadio actual mantiene a los ciudadanos más informados, incluso de sus derechos.

\section{PALABRAS CLAVE}

Ciudadanía, democracia, educación, globalización, identidad,

\section{ABSTRACT}

This article reflects on the meanings of citizenship and democracy and the role education plays in building democracy and identity in Latin America, a historically unequal society. Be a citizen and live in a democracy is to exercise the right to vote. Globalization as currently maintains more informed citizens, including their rights.

\footnotetext{
* El presente artículo forma parte de una investigación denominada "Soberanía, fronteras e integración amazónica", cuyo marco teórico se centra en la discusión actual de la soberanía, como un elemento del Estado, pues la globalización ha llevado a conformar ciudadanos globales que cada vez más se identifican con valores, no necesariamente propios de los latinoamericanos. ** Abogada, Magíster en Análisis de Problemas Políticos Económicos e Internacionales Contemporáneos, Candidata a doctora en la Universidad de Valladolid. Profesora universitaria, consultora.
} 


\section{KEY-WORDS}

Citizenship, democracy, education, globalization, identity.

\section{SUMÁRIO}

Este artigo reflete sobre os significados da cidadania e da democracia e o papel que desempenha a educação na construção da democracia e da identidade na América Latina, uma sociedade historicamente desigual. Ser cidadão e viver em uma democracia é mais que exercer o direito de votar. A globalização atual mantem os cidadãos mais informados, incluindo os seus direitos.

\section{PALAVRAS-CHAVE}

Cidadania, democracia, educação, globalização, identidade.

\section{PROBLEMA DE INVESTIGACIÓN}

El presente artículo forma parte de una investigación denominada "Soberanía, fronteras e integración amazónica", cuyo marco teórico se centra en la discusión actual de la soberanía, como un elemento del Estado, pues la globalización ha llevado a conformar ciudadanos globales que cada vez más se identifican con valores, no necesariamente propios de los latinoamericanos. La propuesta de Stephen Krasner, denominada de "la soberanía compartida", es sugerente y podría representar un peligro para los Estados de América Latina si no se construye ciudadanía, democracia e identidad. Este es problema central para el cual una educación basada en otros valores podría desempeñar un papel fundamental.

\section{METODOLOGÍA}

El enfoque del estudio sigue los lineamientos de la investigación social reflexiva. Dos son las consideraciones básicas que pueden presentarse como explicación desde esta perspectiva metodológica para desarrollar este trabajo. La investigación social reflexiva, como enfoque metodológico, fue esbozada, hace dos décadas, por Enrique Dussel (2000), Aníbal Quijano (2000), Pablo González Casanova (1998) y desarrollada por Lander (1996) y Mignolo (1996), entre otros, como herramienta para abordar la problemática política, económica y cultural en América Latina.

Este enfoque propone una forma reflexiva de asumir la investigación y, básicamente, la metodología como oficio de navegación, distinta de los rígidos encuadramientos y consabidos derroteros que han impuesto desde la academia los modos tradicionales de investigar centrados en un enfoque único y privilegiado para analizar una realidad compleja y cambiante, como la de América Latina.

El segundo aspecto puede ser formulado de la siguiente manera: por enfoque social reflexivo se entiende en este trabajo como la metodología que no reduce la realidad a lo que existe. La realidad, como quiera que se la conciba, es considerada por el enfoque social como un campo de posibilidades. En esta tarea los mencionados autores inicialmente citados han colaborado o se han apoyado en el trabajo de analistas que han trabajado en América Latina como Boaventura de Sousa; en África, como Inmanuel Wallerstein, o en el Medio Oriente, como Edward Said. Estos autores 
sugieren que la cientificidad de una investigación no reside ni en las técnicas ni en las cuantificaciones que a partir de ellas se hagan; más bien depende estrechamente del tipo de preguntas con las que se trata de ordenar una realidad que no está ordenada ni se presta a ser fácilmente aprehendida.

Es preciso subrayar que el mundo social (es decir, el mundo político, económico, de relaciones internacionales, entre otras] es un universo no solo objetivo sino preinterpretado por los agentes sociales, de tal manera que las ciencias sociales siempre tienen que hacer las cuentas con una doble hermenéutica básica: interpretar mundos sociales ya interpretados.

\section{INTRODUCcIÓN}

Ciudadanía y democracia son dos términos que están unidos dialécticamente. En los Estados latinoamericanos el espacio de la ciudadanía se presenta históricamente complejo, pues la democracia ha sido frágil y precaria desde el surgimiento como Estados independientes. La globalización plantea inmensos desafíos e interrogantes pues si la ciudadanía no pudo consolidarse en los términos de la modernidad, actualmente, en medio de una aparente despolitización de la sociedad, no resulta fácil entender de qué manera los movimientos sociales han tenido un protagonismo importante en esta parte del mundo.

El tema es de interés porque a partir de los años noventa, en el seno de los Estados territoriales latinoamericanos, se presentaron hechos que alteraron notoriamente las relaciones internacionales que hasta entonces había conocido la región.

Este corto ensayo pretende exponer algunos interrogantes que surgen al respecto, partiendo de la definición de ciudadanía, su desarrollo en la llamada Era de la Información, así como su relación con la democracia y la educación. Si se afirma que la globalización lleva implícita la formación de "ciudadanos globales", ¿cuál es la percepción que los latinoamericanos tienen del concepto de ciudadanía? ¿Qué papel han desempeñado los ciudadanos en hechos como los ocurridos en Ecuador, Venezuela o Bolivia, que llevaron al traste los modelos tradicionales de democracia representativa? ¿Qué función tiene la educación en la formación de valores globales?

\section{UNA NOCIÓN DE CIUDADANÍA EN EL CONTEXTO GLOBAL}

Hay muchos estudios de ciudadanía que parten de definiciones del término influidas por enfoques políticos. Para efectos de este escrito se adopta la definición de Marshall expuesta en su clásico ensayo "Ciudadanía y clase social", escrito en 1950, según el cual,

La ciudadanía es un status que se otorga a los que son miembros de pleno derecho de una comunidad. Todos los que poseen ese status son iguales en lo que se refiere a los derechos y deberes que implica. No hay principio universal que determine cuáles deben ser estos derechos y deberes, pero las sociedades donde la ciudadanía es una institución en desarrollo crean una imagen de la ciudadanía ideal en relación con la cual puede medirse el éxito y hacia la cual pueden dirigirse las aspiraciones. El avance en el camino así trazado es un impulso hacia una medida más completa de la igualdad, un enriquecimiento del contenido del que está 
hecho ese status y un aumento del número de aquellos a los que se les otorga (Marshall, 1997).

Modernamente el término de ciudadanía empezó a ser desarrollado en la Inglaterra del siglo XVI. Se trata, pues, de una institución propia del sistema capitalista que, como lo señala este autor, "es un sistema no de igualdad, sino de desigualdad" (p. 312). Si bien hoy nos encontramos en otro estadio del capitalismo, su esencia es la misma. La desigualdad no solo se observa en lo local sino también en el ámbito internacional: ni los ciudadanos ni los Estados son iguales. La igualdad, hasta ahora, sigue siendo una ficción legal.

La definición de Marshall corresponde a la sociedad en la que vivió este sociólogo británico, en la cual, terminada la Segunda Guerra Mundial, existía cierto optimismo colectivo y una necesidad de pregonar el Estado de bienestar. En la presente etapa de la globalización, en la posguerra fría, el asunto de la ciudadanía nuevamente cobra interés, precisamente por el hecho de haberse debilitado el concepto de soberanía vatteliana que primó hasta comenzado el siglo XXI, definida como "la exclusión de fuentes externas de autoridad tanto de jure como de facto. Dentro de sus propios límites, el Estado tiene un monopolio sobre la toma de decisiones autorizadas. En el nivel internacional esto implica que los Estados siguen la regla de la no intervención en los asuntos internos de otros" (Krasner, 2001, p. 4). Actualmente, las personas, aun sin tener acceso físico a espacios como el europeo, lo hacen de manera virtual y adquieren valores que les hacen pensar que son ciudadanos del mundo. Los problemas inducen igualmente al ciudadano a pensar de manera global. Vieira (1998) acertadamente lo señala:

Cuestiones como las relativas a producción, comercio, capital financiero, migraciones, pobreza, daños ambientales, desempleo, informatización, telecomunicaciones, en fin, las grandes cuestiones económicas, sociales, ecológicas y políticas, dejaron de ser solo nacionales para tornarse transnacionales. Es en este contexto que nace hoy el concepto de ciudadano del mundo, de ciudadanía planetaria, que viene siendo paulatinamente construido por la sociedad civil de todos los países en contraposición al poder político del Estado y al poder económico del mercado (p. 6).

Específicamente, este momento del desarrollo capitalista se ha dado en llamar también la era de la información. Castells la define como: "Transformación histórica multidimensional definida por la transformación del sistema productivo, del sistema organizativo, del sistema cultural y del sistema institucional sobre la base de una revolución tecnológica que no es la causa pero sí el soporte indispensable" (Castells, 2003, p. 19]. En tales términos, la tecnología es fundamental pero no es la causa ni el efecto: es el medio que permite que ciertas actividades puedan "funcionar como unidad de tiempo real a escala planetaria" (p. 19). Este fenómeno permitió articular a todas las regiones del planeta y globalizar la economía, la ciencia, la cultura, la tecnología, el Derecho, la información y, como lo anota Castells, hasta el crimen organizado. Todo ello se articula a través de redes.

Sin embargo, independientemente de la forma como se manifiesta la globalización actual, los temas de la democracia y de la igualdad están presentes en la cotidianidad 
política como objetivos a alcanzar, aún sin precisar su contenido. Estos temas se convierten en comodines de los discursos políticos, en fantasmas que persiguen a las sociedades pero que no logramos ver, alcanzar, conocer y gozar.

El hecho de sentirse ciudadano del mundo lleva implícita cierta sensación de igualdad, cosa que de alguna manera es cierta, toda vez que la moda, la información, los gustos colectivos y en general el consumo están moldeados y uniformados gracias a que la mass media hace sentir tal sensación y oculta las diferencias de fondo. Esto no es nuevo. Marcuse lo había percibido en los años cincuenta del siglo pasado, y lo consignó en su texto "El hombre unidimensional", en referencia a las clases sociales.

Si el trabajador y su jefe se divierten con el mismo programa de televisión y visitan los mismos lugares de recreo, si la mecanógrafa se viste tan elegantemente como la hija de su jefe, si el negro tiene un Cadillac, si todos leen el mismo periódico, esta asimilación indica, no la desaparición de las clases, sino la medida en que las necesidades y satisfacciones que sirven para la preservación del "sistema establecido" son compartidas por la población subyacente (Marcuse, 1997, p. 16).

Para un europeo su sentido de pertenencia al mundo y, por consiguiente, su sentido de ciudadanía, difiere del que abriga un africano o un latinoamericano, aunque estos últimos se "sientan iguales" porque, teóricamente, pueden consumir lo mismo que los ciudadanos del primer mundo. Precisamente, por las anteriores razones, sigue siendo válida la definición de Marshall según la cual todos los que poseen ese estatus son iguales.

Lo que varía, entonces, es el ejercicio de la ciudadanía y en este sentido el cambio es notable. Como lo anota Lechner (1999),

El redimensionamiento (sic) de la esfera político-estatal ha de afectar la noción de ciudadanía. Cuando el Estado pierde su aura de instancia sacrosanta, cuando el sistema político pierde su centralidad jerárquica, cuando la acción política desborda tanto el marco nacional como el institucional, cuando el discurso político ya no escenifica las dinámicas de la convivencia social ni ofrece esquemas de interpretación, en fin, cuando cambia el ámbito político cambia el ejercicio de la ciudadanía.

La ciudadanía sigue siendo la titular de los derechos pero el ejercicio de la ciudadanía se ha transformado por efecto de la globalización actual.

El sentido de igualdad, que se desprende de los conceptos democracia y ciudadanía, es un objetivo a alcanzar por los ciudadanos del mundo. La igualdad real no existe, sigue figurando en la esfera del deber ser.

El concepto de ciudadanía está extendido y su ejercicio se presenta de dos maneras,

Por un lado, una ciudadanía que puede denominarse instrumental, por cuanto considera a la política como algo ajeno y, no obstante, se dirige al sistema político en tanto (sic) solución a los problemas sociales. No pretende participar en la toma de decisiones ni moldear la marcha del país. Al discurso abstracto opone su mundo concreto 
y reclama una gestión eficiente a favor del bienestar de la gente. Lo que cuenta son los servicios tangibles que presta. Dicho de modo esquemático: la "ciudadanía instrumental" descree de la política y cree en la administración (particularmente la municipal) (Lechner, 1999).

Este ciudadano es el más común. Una manifestación de ello es el clientelismo. En una de sus formas, la gente apoya si se ofrece algo a cambio: cargos públicos, becas, arreglo de vías, entre otros. El abstencionismo, en sentido contrario, también puede ser una manifestación del mismo fenómeno: "no voto, no participo, que decidan otros porque yo no recibo nada a cambio". En ambos casos no se participa en discusiones y la política sigue siendo un asunto de políticos.

La actual globalización también ocasiona la pérdida del sentido de identidad de los pueblos. Si, en los términos de Marcuse, las sociedades no están inmóviles y la identidad es igualmente un proceso infinito de construcción, la actual globalización excluye necesariamente la identidad de los pueblos. Las culturas latinoamericanas y las del mundo entero estarían condenadas a desaparecer para dar vía a una sociedad unidimensional.

Hay otra forma de ciudadanía. Como afirma Lechner (1999), "parece emerger lo que puede llamarse una 'ciudadanía política'. El segundo tipo de ciudadanía se refiere no tanto a la 'política institucionalizada' en el sistema político como a la acción colectiva de los propios ciudadanos". Es una ciudadanía que emerge en Latinoamérica y en el mundo como una respuesta, quizás desesperada, a las grandes injusticias.

Sin embargo, a pesar de los cambios operados en el mundo en los últimos lustros, el concepto clásico de ciudadanía sigue siendo válido, como un deber ser, en la medida en que la búsqueda de la igualdad real es un objetivo a alcanzar. Lo que ha cambiado es el ejercicio y el espacio en el que se ejerce la ciudadanía, pues, por lo menos en Latinoamérica, existe una tendencia a conformar una ciudadanía política activa.

\section{ESCOLLOS Y PARADOJAS DE LA CONSTRUCCIÓN DE CIUDADANÍA EN AMÉRICA LATINA}

Lastres históricos como la falta de identidad, la desigualdad, el clientelismo, el conservadurismo ideológico, los autoritarismos, el racismo encubierto, la fragilidad de la institucionalidad política, la falta de autonomía y desarrollo de la sociedad civil han caracterizado históricamente a la sociedad latinoamericana. Todos estos problemas han impedido la consolidación de una ciudadanía política activa y la construcción de un espacio para la vida democrática. Por eso nuestra ciudadanía es débil.

Los términos ciudadanía e igualdad inducen a reflexionar sobre otro aspecto: la identidad, que, según Castells (2003), "es el proceso por el cual los actores sociales construyen el sentido de su acción atendiendo a un atributo cultural co conjunto articulado de atributos culturales] al que se da prioridad sobre otras fuentes posibles de sentido de la acción" (p. 22). Las identidades de la globalización latinoamericana no están fundadas en tradiciones históricas o culturales. Aparecen unas identidades individuales, fragmentadas y construidas, que giran alrededor de aspectos familiares, territoriales, religiosos, étnicos o de sexo. 
La construcción de democracia, de identidad y de ciudadanía ha encontrado innumerables obstáculos en América Latina. Si bien la lengua constituye una expresión de identidad latinoamericana, por razones históricas, geográficas, económicas y políticas no existen Estados-nación en el sentido europeo. A cambio de ello las nuevas repúblicas se constituyeron como Estados territoriales, fragmentados social, cultural y políticamente. Sus habitantes no poseen un sentido de identidad como latinoamericanos. Mientras en Europa la ciudadanía surgió como manifestación de la identidad de sus pueblos, en Latinoamérica se impuso el concepto de espaldas a las realidades sociales, no pocas veces obedeciendo a intereses mezquinos e individualistas.

José Martí propuso en "Nuestra América" que estos pueblos se reconocieran, pues así podrían establecer y resolver mancomunadamente los problemas comunes que les aquejaban. De esta manera, a partir del encuentro con su identidad, las jóvenes repúblicas se insertarían en el mundo (Martí, 1972). Lejos de lo que pensó este americanista, tal encuentro fue una quimera. Por ello, resulta paradójico hablar de ciudadanía global cuando no se constituyó una ciudadanía latinoamericana que se reconociera. Este es el primer obstáculo para la construcción de una ciudadanía activa puesto que la idea de igualdad en la que se centra este concepto supone una relación con otro y cierto sentido de identidad.

En el actual contexto global la construcción de identidades individuales, étnicas y territoriales va en contravía del sentido clásico de ciudadanía y carece de un sentido colectivo y de pertenencia. Como señala Sonia Fleury (2003),

La fragmentación de la sociedad en grupos y culturas polarizadas, más que una expresión de tolerancia y pluralismo en un contexto posmoderno [...] pasa a ser un indicador de la pérdida de la dimensión colectiva, identificada como la comunidad nacional de los ciudadanos. Esto ocurre, ya sea porque tal condición de pertenencia se niega a aquellos que sufren de diferentes formas de exclusión, o por el desencanto con la política, restringida a su dimensión institucional (p. 130).

Así mismo, los valores de la globalización giran alrededor del consumo y en consecuencia el mercado se teologizó y "la construcción de las identidades pasa a ser producto de la sociedad y del mercado, un bien a ser consumido, una identidad colectiva volátil, normada por patrones de consumo insatisfechos" (Fleury, 2003, p. 130). En este continente coexisten muchos mundos, no fue posible construir una identidad colectiva y lo que hoy se pretende presentar como ciudadanía parte de valores individualistas que niegan las culturas autóctonas, desconocen y excluyen al otro, admiten los valores que impone la cultura dominante del mercado.

En realidad, la percepción de la democracia en la población latinoamericana se identifica generalmente solo con las elecciones, mientras a otros aspectos de la ciudadanía se les concede menor importancia. Al respecto, es ilustrativo el trabajo que presenta la Corporación Latinobarómetro (2008), según la cual, en América Latina, "La ciudadanía está basada en el voto, mientras los impuestos y las leyes están en un segundo lugar bastante lejos. Disminuye el impuesto como componente de la ciudadanía, lo que es congruente con la disminución de la moral impositiva" (p. 104). Esta entidad midió en los años 2007 y 2008 ciertas características del ciudadano que valoran las personas en América Latina. 
A los entrevistados se les preguntó cuáles acciones no pueden dejar de hacer los ciudadanos si quieren ser considerados como tales, por ejemplo, votar, pagar impuestos, obedecer las leyes, ayudar a las personas que están peor que uno, participar en organizaciones sociales, cumplir con el servicio militar, elegir productos de valor ambiental responsable. Los resultados de la encuesta aparecen en el cuadro que acompaña estas notas y son bien dicientes.

MAYOR O MENOR IMPORTANCIA DE LAS CARACTERÍSTICAS DEL CIUDADANO

\begin{tabular}{|l|r|r|}
\hline \multicolumn{1}{|c|}{ Característica } & \multicolumn{1}{|c|}{$\mathbf{2 0 0 7}$} & \multicolumn{2}{c|}{$\mathbf{2 0 0 8}$} \\
\hline Votar & 71 & 72 \\
\hline Pagar impuestos & 52 & 49 \\
\hline Obedecer las leyes & 48 & 45 \\
\hline Ayudar a los que están peor que uno & 37 & 38 \\
\hline Participar en organizaciones políticas & 15 & 13 \\
\hline Participar en organizaciones sociales & 21 & 20 \\
\hline Cumplir con el servicio militar & 20 & 19 \\
\hline Elegir productos ambientalmente responsables & 19 & 18 \\
\hline
\end{tabular}

Fuente: Corporación Latinobarómetro 2008.

Esta encuesta refleja claramente el sentido de ciudadanía que han interiorizado los latinoamericanos.

Fleury (2003) señala estas contradicciones: "Un público globalizado, propiciado por las tecnologías comunicacionales, pero profundamente dividido en el seno de la estructura social de cada país, permite, al mismo tiempo, y paradójicamente, que la ciudadanía se coloque al nivel mundial y que exista un profundo distanciamiento entre los individuos con relación a la bases territoriales y políticas locales y nacionales" (p. 130). Eso querría decir que la sociedad latinoamericana se globalizó y que se identifica con los valores de consumo pero en el seno de los Estados territoriales no logra unirse.

Castells (2003) explica acertadamente que la "globalización, en su encarnación actual del capitalismo informacional desregulado y competitivo, supera a los Estados, pero se articula a los segmentos dinámicos de las sociedades de todo el planeta, al tiempo que desconecta y margina aquellos que no tienen otro valor que el de su vida" (p. 22). En la profundidad de la selva amazónica puede encontrarse un grupo étnico que no habla castellano o portugués pero que observa la transmisión por vía satelital de la final del Campeonato Mundial de Fútbol y toma partido por un equipo europeo.

De acuerdo con la definición de Marshall, cabe preguntar: équiénes son "los miembros de pleno derecho" de Latinoamérica? En la globalización no se han eliminado las asimetrías sociales; incluso la pobreza ha aumentado, como lo muestran los estudios de la Cepal. De acuerdo con esta entidad,

en el 2005 el 39,8\% de la población de la región vivía en situación de pobreza, y el 15,4\% de la población era extremadamente pobre o indigente. Así, los pobres sumaban 209 millones en total, de los 
cuales 81 millones eran indigentes [...]. Por primera vez luego de 25 años, la incidencia de la pobreza es similar a las cifras anteriores a la crisis de la deuda, pero representa 70 millones de habitantes adicionales debido al crecimiento demográfico. Se espera que en el 2006 disminuya el porcentaje de pobres en poco más de un punto porcentual, a 38,5\%, y el porcentaje de indigentes en alrededor de medio punto porcentual, a 14,7\%. Si se logran estos resultados, el número de pobres e indigentes disminuiría nuevamente, a 205 millones y 79 millones, respectivamente. (Cepal, 2007)

Otros estudios, como el de la Corporsción Latinobarómetro (2008), expresan: "Una de las características de América Latina ha sido históricamente la acentuada inequidad de la distribución del ingreso, así como su falta de flexibilidad ante el cambio. Esta desigualdad se mantuvo invariable durante la década de los noventa, empeorando inclusive a comienzos de la presente década" (p. 4). Por su parte la Unesco manifestó: "La región de América Latina y el Caribe es la más inequitativa del planeta. Las diferencias entre quienes tienen más y quienes tienen menos, lejos de reducirse, se acrecientan día a día, ejerciendo un efecto negativo en todos los ámbitos de la vida, incluida la educación" (Unesco, 2011).

Obviamente, la inequidad provoca exclusión social. Los latinoamericanos no tienen plenos derechos, no son iguales en lo que se refiere a derechos y obligaciones. En América Latina impera un modelo caracterizado por asimetrías sociales y exclusión, lo cual no permite a sus miembros gozar de plenos derechos.

América Latina enfrenta la globalización actual en un proceso de crisis. Allí, donde impera un atraso tecnológico, una brecha tecnológica muy grande, no pueden existir las condiciones indispensables para esa globalización. "La inequidad no solo conspira contra la reducción de la pobreza, sino que se transforma en una restricción al propio proceso de conocimiento", manifestó José Luis Machinea, representante de la Cepal (2007).

Otro aspecto problemático está relacionado con el espacio para ejercer la ciudadanía. Al respecto, Pérez Baltodano (1970), concluye que la debilidad de la opinión pública latinoamericana obedece a que no se crearon espacios públicos para discutir los problemas de interés general. El autor utiliza categorías habermasianas como "espacio territorial" o "espacio público". En los años setenta del siglo pasado, Habermas popularizó esta categoría de análisis político para afirmar que es ella el lugar de todos los ciudadanos, donde se juntan para formular una opinión pública que permite presionar al Estado, y al mismo tiempo es el espacio para discutir las libertades, la cultura y la sociedad. Quizás en Latinoamérica, por las razones históricas anotadas, no había existido una opinión pública fuerte.

La pobreza y la exclusión distorsionan el sentido de la democracia. Las personas, si tienen que elegir entre economía y democracia, optan por la primera. Es lógico, la gente desea resolver los problemas de sobrevivencia antes que pensar en participación y, en general, en política. Como lo expresa el informe de la Corporación Latinobarómetro (2008), "hay amplia evidencia de que el significado de la democracia en América Latina tiene un componente económico que no tuvieron otras democracias en otras partes del mundo cuando surgieron" (p. 9). Por esa razón se insiste en 
que los conceptos de democracia y ciudadanía no significan lo mismo para todos ni tienen igual trascendencia en todas las regiones. La pobreza no permite pensar en democracia, y así no interesa ser ciudadano sino sobrevivir.

Otros datos también revelan una fuerte asimetría entre los potenciales ciudadanos del mundo y una contradicción entre los procesos de globalización y las condiciones para la globalización, lo cual provoca crisis. En tales condiciones, ¿cómo se integran estos millones de seres a la sociedad de la información? ¿Se continuará la construcción de la sociedad sobre la exclusión? ¿Cómo se está construyendo la ciudadanía?

Como afirma Lechner (1999),

en realidad el principio de igualdad que rige el ámbito de la ciudadanía (una persona, un voto] puede ser socavado por las desigualdades sociales. Las distancias sociales en ingresos y educación tienden a conllevar una desigualdad de los recursos linformación, medios de expresión y organización, tiempo y dinero], que exige la participación política y, en concreto, la electoral (p. 12).

Las anteriores reflexiones nos ubican en un contexto en el que democracia y ciudadanía se distorsionan en razón de unas realidades diferentes a las de los habitantes del primer mundo. Por ello resulta fundamental analizar el papel de la educación en la formación de ciudadanía y de movimientos sociales, concretamente en el caso de América Latina.

\section{EDUCACIÓN, CIUDADANÍA Y MOVIMIENTOS SOCIALES EN LATINOAMÉRICA}

La educación no le otorga necesariamente el estatus de ciudadano a una persona. Sin embargo, cuando un Estado eleva a precepto constitucional el derecho universal a la educación y lo declara obligatorio, implícitamente se está aceptando un elemento importante de la ciudadanía: la igualdad. Si se trata de un niño, potencialmente se trata de un ciudadano; si es un adulto, tendrá además la posibilidad de conocer sus derechos y obligaciones. Como lo indica Marshall (1997),

El derecho a la educación es un genuino derecho social de ciudadanía, porque el objetivo último de la educación en la infancia es crear al futuro adulto. Debe considerarse esencialmente no el derecho del niño a ir a la escuela, sino el derecho del ciudadano adulto a recibir educación [...] La educación es un prerrequisito necesario para la libertad civil (pp. 310, 311).

A lo largo del siglo XX se impuso la necesidad de educar y por esa razón la educación fue adoptada como un indicativo para establecer el grado de desarrollo de los pueblos.

Desde el punto de vista político, "se tomó cada vez más conciencia de que la democracia política precisaba un electorado educado, y que la manufactura científica precisaba trabajadores y técnicos cualificados" (Marshall, 1997, p. 311). De ello se infiere que, a medida que se logra la universalización de la educación, se va conformando una ciudadanía activa. Este presupuesto no ofrece mayor discusión actualmente. Nadie podría discutir esta necesidad. Hoy la humanidad en general ha logrado escolarizarse 
en alto grado, si se compara con siglos anteriores, aunque surgen cuestionamientos de los contenidos y fines de la educación: ¿Para qué se educa? ¿Qué valores transmite la educación? ¿Realmente se educa para una ciudadanía política activa?

Uno de los efectos de la actual globalización se observa en la educación. Como lo expone el profesor Díaz Arenas (2000), "la educación, cada vez más ajena a la pedagogía y convertida en asunto empresarial, logra su pleno cometido si los niños desde que balbucean quedan inmersos en la sociedad de consumo y los adolescentes en estadio avanzado de consumo son decididamente hedonistas" (p. 37). Estos valores son asumidos por los niños y adolescentes de todas las clases sociales. "Los jóvenes, todos obsedidos por ser apuestos, millonarios y renombrados, desprecian entonces a los desheredados por la naturaleza y a los desafortunados sociales" (p. 7). Este fenómeno es común en todos los países.

De otro lado, en la "era de la información" la educación cobra otro valor. Castells (2003) afirma:

Las fuentes de productividad y competitividad en la nueva economía global dependen fundamentalmente de la capacidad de generación de conocimiento y procesamiento eficaz de la información. La cual depende, a su vez, de la capacidad cultural y tecnológica de las personas, empresas y territorios. En la economía informacional, la educación y la innovación se constituyen en fuerzas productivas directas (p. 21).

Este aspecto es fundamental para entender hacia dónde se dirige la educación en la era de la información. No obstante, la sociedad latinoamericana no está preparada para ello.

De acuerdo con el informe de la Unesco, citado por el Ministerio de Educación de Colombia (2006), América Latina, con pocas excepciones, ha universalizado la educación primaria y muestra logros en educación preescolar y superior. El gasto público en la región supera al de África pero está lejos de alcanzar el de los países del primer mundo. "América Latina destina US \$614 de gasto público por alumno de educación primaria. África subsahariana US \$167, mientras que los países desarrollados US \$5.000" (Ministerio de Educacion, 2008) Estas cifras son contundentes. En materia de educación superior la brecha es mayor si se comparan el contexto latinoamericano con el norteamericano o el europeo.

En América Latina existe una heterogeneidad muy grande entre las universidades y además el PIB regional en educación superior es marcadamente inferior. Sin embargo, pese a ello, varios de los instrumentos propuestos como la creación de redes, las prácticas en entidades públicas y privadas, la formación de personal docente se están desarrollando.

En los últimos años la educación ha ejercido un papel crucial en la consolidación de los movimientos sociales latinoamericanos. Como lo afirma el mencionado el estudio sobre la opinión pública adelantado de la Corporación Latinobarómetro [2008],

La permanencia de las desigualdades explica el grado limitado de consolidación que han alcanzado las democracias en América Latina y las dificultades de gobernabilidad, toda vez que ha aumentado el 
nivel de educación en estos 30 años desde la transición y que los ciudadanos cada vez tienen más instrumentos para hacer valer sus derechos" (p. 12).

Lo que ocurre en naciones latinoamericanas como Venezuela, Bolivia y Ecuador es incomprendido en muchos otros espacios. La historia latinoamericana llegó por distintos caminos a la modernidad y, actualmente, a la nueva etapa de la globalización.

Para estos pueblos la educación ha sido,

el instrumento que tienen para protestar cada vez más, ya que tienen más educación para hacer valer sus derechos. Entregar grados mayores de democracia, con la educación y mejores niveles de vida, es un boomerang para los gobernantes que se les vuelve en contra con mayores demandas. Ser más democrático resulta a veces políticamente no rentable (p.12).

En América Latina los movimientos sociales populares buscan erradicar las desigualdades, lo cual ha permitido llevar al poder líderes que han propuesto reformas revolucionarias desde el Estado, a partir de cambios constitucionales. "Paradojalmente, los países donde mejoran más los indicadores sobre la democracia son los más convulsionados por esa revolución institucional" (pp. 9-10).

En los años noventa irrumpió en Venezuela un fuerte movimiento social, el "Caracazo", que fue el inicio de una oleada de movimientos desplegados en la región. En Ecuador, Bolivia, Argentina también se han producido movilizaciones sociales que han terminado con la salida abrupta del poder de mandatarios corruptos. En esos países se han presentado grandes tensiones sociales ocasionadas por fuerzas opuestas a los cambios. En tres de estos países se conformaron asambleas constituyentes que expidieron nuevas constituciones con la pretensión de refundar los Estados.

En Bolivia, en julio de 2007, en un momento de graves convulsiones sociales, el gobierno lanzó un programa para erradicar el analfabetismo. Pese a la alta ingobernabilidad que reinaba entonces en la nación, el Ministerio de Educación y Culturas (2008) anunció que para diciembre de 2008 se habría erradicado el analfabetismo del territorio patrio, tal como efectivamente ocurrió (Ministerio de Educacion y Culturas de Bolivia, 2008). En ese país los movimientos ciudadanos han librado luchas muy importantes contra la privatización de los recursos naturales y por el derecho a la vivienda. Quizás la globalización brinda oportunidades a la ciudadanía para salir del "encierro territorial nacionalista" y revalorar el espacio local.

Es diciente que países como Venezuela, Bolivia, Ecuador, Nicaragua y, obviamente, Cuba, criticados permanentemente por la forma como asumen temas como la democracia, la libertad y, por supuesto, la economía, sea la educación un renglón prioritario dentro de las políticas públicas de Estado y en el que muestran buenos resultados. En el último informe de la Unesco sobre el seguimiento a la EPT en el mundo dice, con relación al Plan Iberoamericano de Alfabetización y Educación Básica de Personas Jóvenes y Adultas (PIA):

Iniciado en 2007, este plan tiene por objetivo conseguir la alfabetización de toda la población adulta en 2015, impartiendo tres años de educación 
básica a 34 millones de adultos analfabetos. También pueden pretender a recibir un apoyo educativo 110 millones de personas jóvenes y adultas que, en plena edad activa, no han finalizado sus estudios de primaria y que son consideradas analfabetos funcionales. En 2008, el programa "Yo, sí puedo", creado en 2003 por iniciativa del gobierno de Cuba, funcionaba en 12 de los 19 países de América Latina. Este programa se ha integrado en estrategias más vastas encaminadas a conseguir la alfabetización universal de la población adulta en el Estado Plurinacional de Bolivia, Ecuador, Nicaragua, Panamá y la República Bolivariana de Venezuela (Unesco, 2011, p. 9).

Sin embargo este es el primer paso. El nuevo reto es una educación con calidad y con valores. La práctica de la ciudadanía depende de la reactivación de la esfera pública, donde los individuos pueden actuar colectivamente e involucrarse en deliberaciones sobre todos los asuntos que afectan a la comunidad. Esta práctica es esencial para la construcción de una identidad política basada en valores de solidaridad, autonomía y reconocimiento de la diferencia. La ciudadanía participativa es también esencial para alcanzar la acción política efectiva, que habilite a cada individuo para tener algún impacto en las decisiones que afectan el bienestar general. Finalmente, la práctica de la ciudadanía democrática es crucial para expandir la opinión pública y cotejar nuestros juicios; en ese sentido, es un elemento esencial en la constitución de una cultura política vibrante y democrática (Vieira, 1998, p. 6).

La construcción de una ciudadanía activa es posible si la educación y los educadores forman en otros valores, diferentes al consumo, tales como la solidaridad, el respeto hacia el otro, incluida la naturaleza, y la búsqueda de un sentido de pertenencia.

\section{CONCLUSIÓN}

Si bien por razones históricas y políticas no se ha construido en América Latina una ciudadanía activa, con sentido de pertenencia e identidad, la educación y los educadores se encuentran ante el reto de formar ciudadanos con valores diferentes al consumo. Quizás sea una utopía, en una sociedad mercurial como la actual, pero es un deber ético buscar otro mundo posible. 


\section{BIBLIOgRAFÍA}

Aristóteles (1978). La política. Madrid: Colección Austral. Espasa Calpe S.A.

Boni Aristizabal, Alejandra y Pérez-Foguet, Agustí (coords.) (2006). "Instrumentos para el impulso de la educación para el desarrollo de la universidad". En Construir la ciudadanía global desde la universidad: Propuestas pedagógicas para la introducción de la educación para el desarrollo en las enseñanzas científico-técnicas. Barcelona: Intermón Oxfam.

Castells, M. (2003). "Panorama de la era de la información en América Latina ¿Es sostenible la globalización de América Latina?". En Debates con Manuel Castells. México: Fondo de Cultura Económica.

Comisión Económica para América Latina (CEPAL) (2007). "Los objetivos de desarrollo del Milenio y los desafíos para América". Disponible en: http://www. eclac. org/publicaciones/xml/0/30260/ObjetivosdelMilenio_LCW143.pdf (recuperado el 29 de enero de 2009).

Corporación Latinobarómetro [2008). Latinobarómetro 2008. Opinión pública latinoamericana. Santiago de Chile. Disponible en: http://www. latinobarometro. org/docs/ INFORME_LATINOBAROMETRO_2008.pdf (recuperado el 28 de noviembre de 2008).

Díaz Arenas, P. F. (2000). Valores y héroes de la globalidad. Bogotá: Editorial El Búho.

Dussel, E. (2000). Hacia una filosofía política crítica. Madrid: Descleé de Broker.

Fleury, S. [2003]. Legitimidad, Estado y cultura política. México: Fondo de Cultura Económica.

González Casanova, P. (1998). "Reestructuración de las Ciencias Sociales: hacia un nuevo paradigma". En Briceño-León, R. y Heinz, S. (Eds.), Pueblo, época y desarrollo: la sociología en América Latina. Caracas: Nueva Sociedad.

Krasner, S. (2001). "La soberanía perdurable", Colombia Internacional. No. 53, pp. 25-42.

La Jornada UNAM (23 de septiembre de 2007). "Cepal: la pobreza y desigualdad en AL no corresponden al grado de desarrollo". Disponible en: http://www. jornada. unam. $\mathrm{mx} / 2007 / 09 / 23 /$ index. php?section=economia\&article $=024 \mathrm{n} 1 \mathrm{eco}$.

Lander, E. (1996). La colonialidad del saber: eurocentrismo y ciencias sociales. Buenos Aires: Clacso.

Lechner, N. (noviembre de 1999). "Las condiciones sociopolíticas de la ciudadanía. Conferencia de clausura del IX Curso Interamericano de elecciones y democracia" (Instituto Interamericano de Derecho Humanos CAPEL). Disponible en: http://www. desarrollohumano.cl/textos/Extension/Discursos/capel.pdf (recuperado el 7 de agosto de 2001).

(1999). Las condiciones sociopolíticas de la ciudadanía". Conferencia de clausura del IX Curso Interamericano de Elecciones y Democracia. CAPEL. México. 
Marcuse, H. (1997). El hombre unidimensional. Ensayo sobre la ideología de la sociedad industrial de la sociedad industrial avanzada. Barcelona: Ariel.

Marshall, T. H. (1997). "Ciudadanía y clase social". Disponible en: http://www. reis. cis.es/REISWeb/PDF/REIS_079_13.pdf (recuperado el 7 de agosto de 2011).

Martí, J. (1972]. Nuestra América. Antología. Navarra: Biblioteca General Salvat.

Mignolo, W. (1996). Herencias coloniales y teorías poscoloniales. Caracas: Nueva Sociedad.

Ministerio de Educación de Colombia (26 de noviembre de 2008). "América Latina, positivo avance en educación: ONU". Disponible en: http://www. mineducacion.gov.co/ observatorio/1722/article-177261.html (recuperado el 7 de agosto de 2011).

Ministerio de Educación y Culturas de Bolivia (2008). Programa Nacional de Alfabetización. Disponible en: http://www. minedu. gov. bo/minedu/redirect. do?page=/ pna/sis. html (recuperado el 1 de diciembre de 2008).

Montañez, G. (2001). Razón y pasión del espacio y el territorio. Bogotá: Universidad Nacional de Colombia.

Pérez Baltodano, A. (1997). "Estado, ciudadanía y política social en América Latina" En: Globalización, ciudadanía y política social en América Latina: tensiones y contradicciones. Caracas: Nueva Sociedad.

Quijano, A. (2000). "Colonialidad del Poder, Eurocentrismo y América Latina". En Lander, Edgardo (comp.), La colonialidad del saber: Eurocentrismo y Ciencias Sociales. Buenos Aires: CLACSO.

Unesco (2011). "Educación de calidad para todos y el Proyecto Regional de Educación para América Latina y el Caribe (PRELAC)". Disponible en: http://portal. unesco.org/ geography/es/ev. php-URL_ID=7464\&URL_DO=DO_TOPIC\&URL_SECTION=201. html

(recuperado el 8 de agosto de 2011).

(2011). Informe de seguimiento a EPT en el mundo. Una crisis encubierta: conflictos armados y educación. París: Unesco.

Vieira, L. (1998). "Ciudadanía y control social". En Cunillgram Grau, N. y Bresser Pereira, L. C. (coords.), Lo público no estatal en la reforma del Estado. Argentina: Paidós. 